\title{
Growth and characterization of an epitaxially grown ZnSSe/MnZnSSe distributed Bragg reflector
}

\author{
A. Salokatve, K. Rakennus, P. Uusimaa, and M. Pessa \\ Department of Physics, Tampere University of Technology, P. O. Box 692, FIN-33101 Tampere, Finland
}

T. Aherne, J. P. Doran, J. O'Gorman, and J. Hegarty

Department of Physics, Trinity College, Dublin 2, Ireland

(Received 22 March 1995; accepted for publication 22 May 1995)

\begin{abstract}
A Bragg reflector consisting of a 25-period MnZnSSe/ZnSSE Bragg stack is reported. The II-VI semiconductor structure was grown by molecular beam epitaxy on a GaAs (100) epilayer. Structural characterization of the Bragg reflector was performed with double crystal x-ray diffraction and transmission electron microscopy. These studies indicated that the epitaxial II-VI structure, whose total thickness is about $2150 \mathrm{~nm}$, remains pseudomorphic with the GaAs substrate. The Bragg stack has a maximum reflectance of $81 \%$ at $468 \mathrm{~nm}$. This result shows that fabrication of high reflectance mirrors from epitaxial ZnSe-based II-VI compounds is possible in spite of relatively small refractive index differences between constituent II-VI layers. (C) 1995 American Institute of Physics.
\end{abstract}

Efficient light emitters operating in the green/blue visible spectral range are required for many applications such as optical data storage, biomedicine, undersea communications, and displays. Recently there has been rapid progress in development of blue/green light emitters made from $\mathrm{ZnSe}$ based II-VI compound semiconductors. ${ }^{1,2}$ For example, an important milestone in this field has been the recent demonstration of room temperature, continuous-wave blue emission from II-VI laser diodes. ${ }^{3,4}$ Equally impressive progress has been reported in II-VI light emitting diodes (LEDs), especially for structures that have been grown on $\mathrm{ZnSe}$ substrates. ${ }^{5}$ For optoelectronic applications requiring arrays of emitters (e.g., displays and interconnects) it is well known that many benefits accrue from engineering the emission normal to the epilayer growth, as for example in vertical cavity surface emitting lasers (VCSELs) and, of perhaps more importance for displays, planar surface emitting LEDs. Enhanced emission from planar LEDs can be obtained by strategically placing the light emitting region between high reflectance mirrors. Efficient emission with $>10 \%$ external quantum efficiency by III-V planar LEDs using such microcavity design has been reported recently. ${ }^{6}$ These microcavity LEDs have other advantages over standard planar LEDs such as increased directionality. High reflectance mirrors play a central role in all such advanced VCSEL and LED structures and can be grown either epitaxially or by deposition of dielectric stacks or metals. Epitaxial mirrors are, however, more attractive since sample processing is minimized. In II-VI compound semiconductors fabrication of high quality epitaxial mirrors is especially challenging due to small refractive index differences of compatible constituent mirror materials. These difficulties are compounded by lack of precise data on the optical constants of II-VI materials. In this letter, we report on the growth and characterization of a high reflectance II-VI Bragg mirror consisting of 25 pairs of $\mathrm{ZnSSe} / \mathrm{MnZnSSe}$ where the optical thickness of each layer is $\lambda_{0} / 4$ and the design wavelength $\lambda_{0}$, is $480 \mathrm{~nm}$. We measure a peak reflectance $(R)>81 \%$ at $\lambda=468 \mathrm{~nm}$. This is the high- est reported reflectance of an epitaxially grown II-VI multilayer mirror structure in the blue spectral range. The results auger well for future II-VI vertical cavity structures.

In this work II-VI structures were grown by molecular beam epitaxy (MBE) on epitaxial GaAs (100) buffer layers, which were also grown by MBE. Since our III-V and II-VI MBE reactors are not interconnected, the GaAs buffer layers were coated with $\mathrm{As}_{4}$ prior to transferring the samples from the III-V reactor into the II-VI reactor. The protective $\mathrm{As}_{4}$ layer was subsequently removed in the II-VI reactor by heating the sample to $400{ }^{\circ} \mathrm{C}$. Growth of $\mathrm{ZnSe}$ buffer layers on samples pretreated in this way commenced in a twodimensional fashion as was evident from streaky reflection high-energy electron diffraction (RHEED) patterns immediately after the $\mathrm{Zn}$ and Se effusion cell shutters were opened. Knudsen effusion cells were loaded with elemental Mn, Se, $\mathrm{Zn}$, one cell for each, and two cells with compound $\mathrm{ZnS}$. Beam fluxes were measured with a nude ionization gauge placed in front of the substrate position. The II/VI flux ratio was close to unity. The growth temperature was $270{ }^{\circ} \mathrm{C}$. Prior to Bragg mirror, growth rates and compositions of constituent materials were calibrated by growing individual layers of ZnSSe and MnZnSSe. Epilayer thicknesses were measured using a stylus profilometer, while compositions were calibrated with double crystal x-ray diffraction (DCXRD) and energy dispersive $\mathrm{x}$-ray analysis (EDX).

In order to prevent relaxation of the layers the distributed Bragg reflectors were grown using lattice matched compositions corresponding to $\mathrm{ZnS}_{0.06} \mathrm{Se}_{0.94}$ and $\mathrm{Mn}_{0.19} \mathrm{Zn}_{0.81} \mathrm{~S}_{0.24} \mathrm{Se}_{0.76}$. The Bragg mirrors consisted of 25 $\mathrm{ZnSSe} / \mathrm{MnZnSSe}$ layer pairs with the optical thickness of each layer being $\lambda / 4$, where the design wavelength is 480 $\mathrm{nm}$. The refractive index of $\mathrm{ZnSSe}$ at $\lambda=480 \mathrm{~nm}$ is about 2.77, while that of MnZnSSe is not well known, but was assumed to be 2.65 for the calculation of layer thicknesses. Transfer matrix calculations predict a peak reflectance of $86 \%$ for this structure. The DBR structural quality was characterized by DCXRD, a spectrum of which is shown in Fig. 


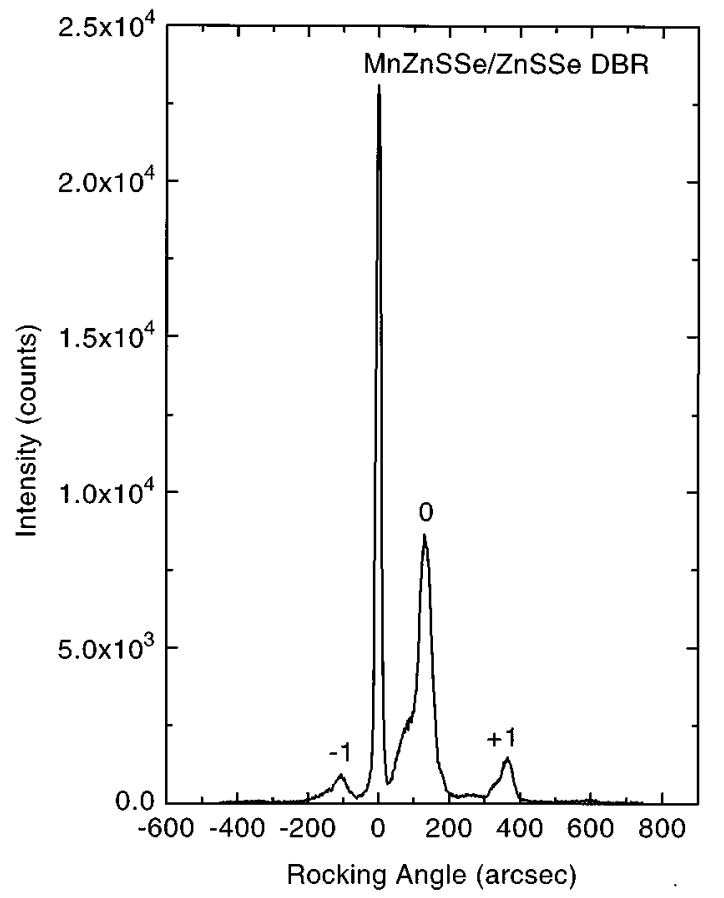

FIG. 1. A DCXRD rocking curve from a 25-period MnZnSSe/ZnSSe DBR sample. Superlattice reflection orders are denoted in the figure.

1. Satellite peaks which are typical of a periodic structure are visible in the DCXRD rocking curve. From the satellite peak separation a periodic structure of thickness $84.5 \mathrm{~nm}$ is calculated. This value is quite close to the designed period of 88.1 $\mathrm{nm}$. The full width at half-maximum (FWHM) of the zero order peak is 43 arcsec which indicates that the structure has remained coherently strained, i.e., no gross relaxation of the structure has taken place.

Cross-sectional samples for transmission electron microscopy (TEM) were prepared using standard methods of mechanical thinning and Ar-ion milling. Figure 2 shows a cross-sectional TEM micrograph from a 25 period Bragg stack. The layer period measured from the micrograph is

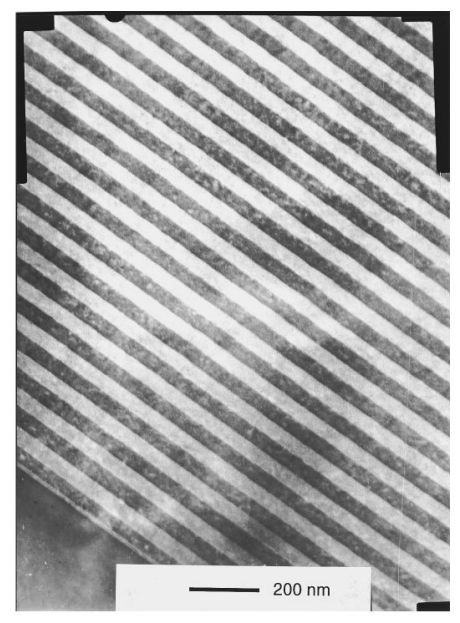

FIG. 2. A cross-sectional TEM photograph from a 25-period DBR sample. The dark layers correspond to MnZnSSe and light layers to $\mathrm{ZnSSe}$, both lattice matched with GaAs.

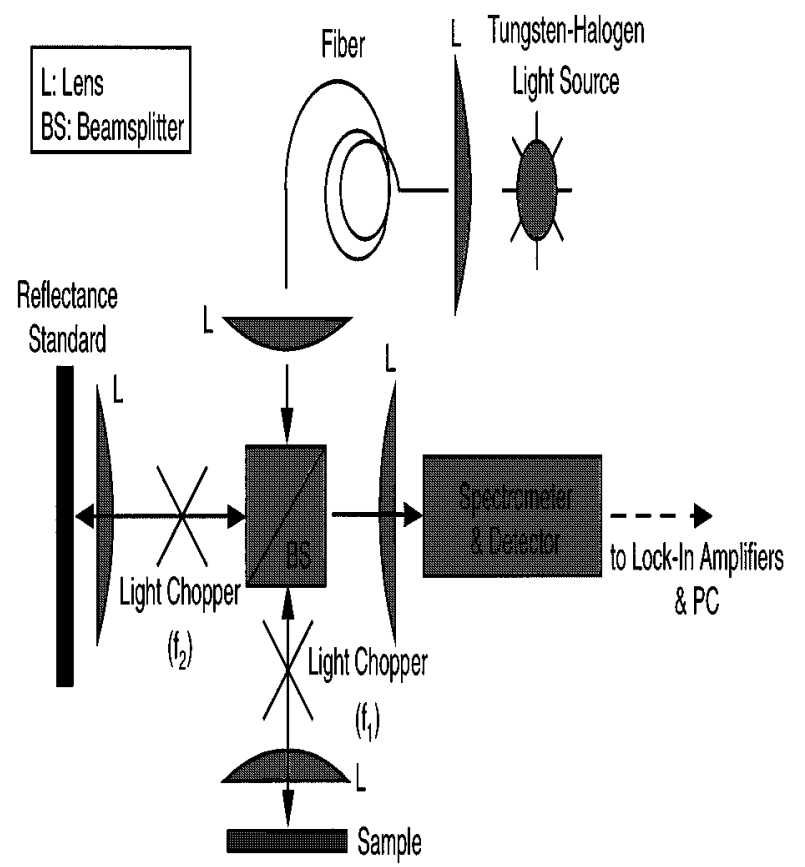

FIG. 3. Double-arm reflectance measurement setup used for the DBR samples.

$85.5 \mathrm{~nm}$, the individual layer thicknesses being 43 and 42.5 $\mathrm{nm}$ for $\mathrm{ZnSSe}$ and MnZnSSe, respectively. It should be noted that the first layers which are close to the GaAs substrate are a little thicker than subsequent layers. This difference may be explained by a slightly lower substrate temperature (and therefore better sticking of atoms at the surface) at the beginning of growth since the high $\mathrm{ZnS}$ and $\mathrm{Mn}$ effusion cell temperatures are expected to increase the substrate temperature when it is exposed to the $\mathrm{ZnS}$ and $\mathrm{Mn}$ fluxes for extended time periods. Some roughness of the interface is visible in the TEM picture. Currently, it is not clear whether this large-scale roughness is real or whether it is caused by the TEM sample preparation. Stacking faults were observed in some areas of the sample, however, most of the sample was free of misfit dislocations, as expected on the basis of close lattice matching of the constituent layers which is corroborated by the width of the DCXRD rocking curve.

Reflectance measurements were performed using a double-arm reflectometer as shown in Fig. 3. White light from a tungsten-halogen source is coupled into the system using a multimode fiber with a $100 \mu \mathrm{m}$ diameter. This double-arm configuration has the advantage of nulling the effect of any noise or drift in the source intensity with time. The reflectance standard used was a commercial mirror exhibiting a certified reflectance $>98 \%$ over the wavelength range of interest. All reflectance spectra were normalized to account for any asymmetry between the two arms of the reflectometer. (The normalization procedure involved placing a second high reflectance mirror at the sample position and taking the ratio of the signals from the two arms. Subsequent reflectance spectra were divided by this normalization spectrum.) The experimental error was determined to be $< \pm 1 \%$. Figure 4 shows a reflectance spectrum taken at normal incidence for a 25-period $\mathrm{ZnSSe} / \mathrm{MnZnSSe}$ distributed 


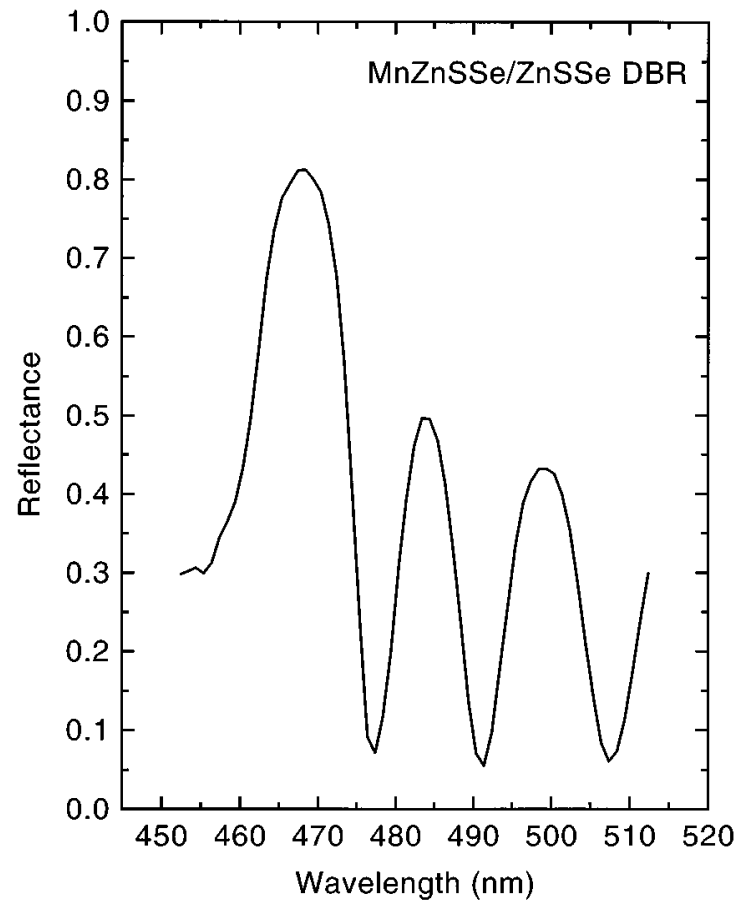

FIG. 4. The reflectance spectrum from a 25-period DBR sample showing a maximum reflectance of over $81 \%$ at $468 \mathrm{~nm}$.

Bragg reflector. A distinct maximum in reflectance of about $81 \%$ is observed at $468 \mathrm{~nm}$. The narrow spectral width of the high reflectance stop band is due to the small refractive index difference between $\mathrm{ZnSSe}$ and MnZnSSe while the absence of oscillations on the short-wavelength side of the stop band is due to absorption in the $\mathrm{ZnSSe}\left(E_{g}=2.736 \mathrm{eV}, \lambda=453 \mathrm{~nm}\right)$ mirror layers. The reflectance value in the short-wavelength region decreases to $\sim 30 \%$ due to this onset of absorption. From calculations based on a transfer matrix method, assuming layer thicknesses as measured by TEM, we have estimated the difference in the average index to be about 0.07 at the central wavelength. The discrepancy between the measured wavelength of peak reflectance and the design wavelength of $480 \mathrm{~nm}$ can be easily explained by uncertainties in both the growth rates and optical constants of the constituent materials. We note that II-VI Bragg mirrors, due to the inherently small refractive index differences between the constituent layers, should be designed for a wavelength that is close to the band gap of the smaller gap material. In this wavelength region the strong dispersion of the refractive index helps to increase the index difference and to obtain high reflectance values.

In summary, we have grown 25-period $\mathrm{ZnSSe} / \mathrm{MnZnSSe}$ distributed Bragg mirrors by molecular beam epitaxy. To the best of our knowledge, these are the first pseudomorphic II-VI Bragg mirrors operating in the blue wavelength region ever fabricated. The Bragg mirrors were characterized with DCXRD, cross-sectional TEM and reflectance measurements. Both DCXRD and TEM characterization show that the structures are lattice matched with GaAs and that no major lattice relaxation by misfit dislocations has taken place. The Bragg mirror reflectance at normal incidence is measured to be $>81 \%$ at $\lambda=468 \mathrm{~nm}$. Such high reflectance epitaxially grown DBR structures will allow growth of planar visible light emitters with enhanced emission efficiency and directionality.

This work was carried out within the European ESPRIT Basic Research Project No. 8447 (SMILES). It was also supported, in part, by The Academy of Finland within a National Materials Research Project (EPIMATTER) and by Optronics, Ireland, the Irish National Initiative in Optoelectronics.

${ }^{1}$ M. Haase, J. Qiu, J. DePuydt, and H. Cheng, Appl. Phys. Lett. 59, 1272 (1991).

${ }^{2}$ H. Jeon, J. Ding, W. Patterson, A. V. Nurmikko, W. Xie, D. C. Grillo, M. Kobayashi, and R. L. Gunshor, Appl. Phys. Lett. 59, 3619 (1991).

${ }^{3}$ N. Nakayama, S. Itoh, K. Nakano, H. Okuyama, M. Ozawa, A. Ishibashi, M. Ikeda, and Y. Mori, Electron. Lett. 29, 1488 (1993).

${ }^{4}$ A. Salokatve, H. Jeon, J. Ding, M. Hovinen, A. V. Nurmikko, D. C. Grillo, Li He, J. Han, Y. Fan, M. Ringle, and R. L. Gunshor, Electron. Lett. 29, $2192(1993)$

${ }^{5}$ D. B. Eason, Z. Yu, W. C. Hughes, C. Boney, J. W. Cook, Jr., J. F. Schetzina, G. Cantwell, and W. C. Harsch, Proceedings of the Seventh Annual Meeting of IEEE Lasers and Electro-Optics Society (IEEE, Boston, 1994), Vol. 1, p. 329.

${ }^{6}$ H. De Neve, J. Blondelle, R. Baets, P. Demeester, P. Van Daele, and G. Borghs, IEEE Photon. Technol. Lett. 7, 287 (1995). 